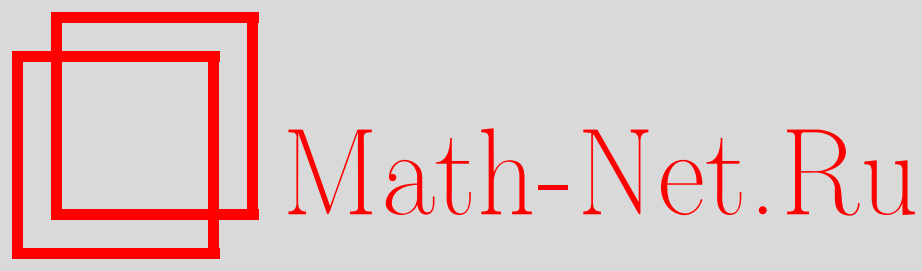

Л. А. Тахтаджян, О вещественных проективных связностях, подходе В.И. Смирнова и решениях уравнения Лиувилля типа черных дыр, ТМФ, 2014, том 181, номер 1, 206-217

DOI: https://doi.org/10.4213/tmf8646

Использование Общероссийского математического портала Math-Net.Ru подразумевает, что вы прочитали и согласны с пользовательским соглашением http: //www.mathnet.ru/rus/agreement

Параметры загрузки:

IP : 54.89 .56 .158

26 апреля 2023 г., 07:19:02

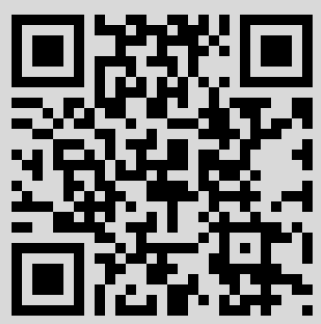




\section{О ВЕЩЕСТВЕННЫХ ПРОЕКТИВНЫХ СВЯЗНОСТЯХ, ПОДХОДЕ В.И. СМИРНОВА И РЕШЕНИЯХ УРАВНЕНИЯ ЛИУВИЛЛЯ ТИПА ЧЕРНЫХ ДЫР}

\footnotetext{
Рассмотрены вещественные проективные связности на римановых поверхностях и отвечающие им решения уравнения Лиувилля. Показано, что эти решения имеют сингулярности специального типа (типа черных дыр) на конечном числе простых аналитических контуров. Подробно разобран случай сферы Римана с четырьмя вещественными проколами, рассмотренный в магистерской диссертации В. И. Смирнова (Петроград, 1918 год).
}

Ключевые слова: униформизация, римановы поверхности, проективные связности, фуксова проективная связность, группа монодромии, уравнение Лиувилля, действие Лиувилля, сингулярные решения.

DOI: $10.4213 / \operatorname{tmf} 8646$

\section{Посвящается моему Учителю Людвигу Дмитриевичу Фаддееву \\ в связи с его восъмидесятилетием}

\section{1. ВВЕДЕНИЕ}

Одной из центральных проблем математики во второй половине XIX - в начале XX века была задача об униформизации римановых поверхностей. Классики, Клейн [1], и Пуанкаре [2], связывали ее с исследованием обыкновенных дифференциальных уравнений 2-го порядка с регулярными особыми точками. Другой подход к задаче об униформизации был предложен Пуанкаре [3]. Он состоит в нахождении полной конформной метрики постоянной отрицательной кривизны и сводится к глобальной разрешимости уравнения Лиувилля, специального нелинейного уравнения в частных производных эллиптического типа на римановой поверхности.

В настоящей работе мы поясняем связи между этими двумя подходами и описываем решения уравнения Лиувилля, отвечающие обыкновенным дифференциальным

${ }^{*}$ Department of Mathematics, Stony Brook University, Stony Brook, NY, USA.

E-mail: leontak@math.sunysb.edu

${ }^{\dagger}$ Международный математический институт им. Л. Эйлера, Санкт-Петербург, Россия 
уравнениям 2-го порядка с вещественной группой монодромии. В современной физической литературе, посвященной уравнению Лиувилля, распространено утверждение о том, что вещественность группы монодромии соответствующего обыкновенного дифференциального уравнения 2-го порядка на римановой поверхности является достаточным условием ее фуксовой униформизации. Однако еще классикам было известно, что это не так, и ими был подробно разобран случай вещественной группы монодромии для соответствующего обыкновенного дифференциального уравнения 2 -го порядка на римановой поверхности рода 0 с проколами. В то же время связь с уравнением Лиувилля ими не была рассмотрена, и здесь мы частично восполняем этот пробел.

Именно, в разделе 2, следуя лекциям [4], мы кратко излагаем теорию проективных связностей на римановой поверхности - инвариантный способ определения соответствующего обыкновенного дифференциального уравнения 2-го порядка с регулярными особыми точками. Следуя [5], [6], мы сообщаем основные результаты, связанные с фуксовой униформизацией, уравнением Лиувилля и комплексной геометрией пространства модулей. В разделе 3, следуя работе [7], мы приводим современную классификацию проективных связностей с вещественной группой монодромии и результаты магистерской диссертации В.И. Смирнова [8] (Петроград, 1918 год). Эта диссертация, опубликованная в [9], [10], была первой работой, где для случая четырех вещественных проколов была приведена полная классификация уравнений с вещественной группой монодромии. В п. 3.2 мы приводим современную интерпретацию результатов В.И. Смирнова. В разделе 4 мы описываем решения уравнения Лиувилля с сингулярностями типа черных дыр, ассоциированные с вещественными проективными связностями. Насколько нам известно, подобные решения ранее не рассматривались.

\section{2. ПРОЕКТИВНЫЕ СВЯЗНОСТИ, УНИФОРМИЗАЦИЯ И УРАВНЕНИЕ ЛИУВИЛЛЯ}

2.1. Проективные связности. Пусть $X_{0}$ - компактная риманова поверхность рода $g$ с отмеченными точками $x_{1}, \ldots, x_{n}$, где $2 g+n-2>0$, и пусть $\left\{U_{\alpha}, z_{\alpha}\right\}-$ комплексно-аналитический атлас с локальными координатами $z_{\alpha}$ и функциями перехода $z_{\alpha}=g_{\alpha \beta}\left(z_{\beta}\right)$ на $U_{\alpha} \cap U_{\beta}$. Обозначим через $X=X_{0} \backslash\left\{x_{1}, \ldots, x_{n}\right\}$ соответствующую риманову поверхность типа $(g, n)$ - поверхность рода $g$ с $n$ проколами. Набор $R=\left\{r_{\alpha}\right\}$, где $r_{\alpha}$ - голоморфная функция на $U_{\alpha} \cap X$, называется (голоморфной) проективной связностъю на $X$, если на каждом из пересечений $U_{\alpha} \cap U_{\beta} \cap X$

$$
r_{\beta}=r_{\alpha} \circ g_{\alpha \beta}\left(g_{\alpha \beta}^{\prime}\right)^{2}+\mathcal{S}\left(g_{\alpha \beta}\right)
$$

где $\mathcal{S}(f)$ - производная Шварца голоморфной функции $f$,

$$
\mathcal{S}(f)=\frac{f^{\prime \prime \prime}}{f^{\prime}}-\frac{3}{2}\left(\frac{f^{\prime \prime}}{f^{\prime}}\right)^{2} .
$$

При этом дополнительно предполагается, что если $x_{i} \in U_{\alpha}$ и $z_{\alpha}\left(x_{i}\right)=0$, то

$$
r_{\alpha}\left(z_{\alpha}\right)=\frac{1}{2 z_{\alpha}^{2}}+O\left(\frac{1}{\left|z_{\alpha}\right|}\right), \quad z_{\alpha} \rightarrow 0 .
$$


Проективные связности образуют аффинное пространство $\mathcal{P}(X)$ над комплексным векторным пространством $\mathcal{Q}(X)$ голоморфных квадратичных дифференциалов на $X$; элементами $\mathcal{Q}(X)$ являются наборы $Q=\left\{q_{\alpha}\right\}$ с законом преобразования

$$
q_{\beta}=q_{\alpha} \circ g_{\alpha \beta}\left(g_{\alpha \beta}^{\prime}\right)^{2}
$$

и дополнительными условиями $q_{\alpha}\left(z_{\alpha}\right)=O\left(\left|z_{\alpha}\right|^{-1}\right)$ при $z_{\alpha} \rightarrow 0$, если $x_{i} \in U_{\alpha}$ и $z_{\alpha}\left(x_{i}\right)=0$. Пространство $\mathcal{Q}(X)$ имеет комплексную размерность $3 g-3+n$. (Подробнее о проективных связностях и квадратичных дифференциалах см. [4] и цитированную там литературу.)

Проективная связность $R$ естественно определяет линейное дифференциальное уравнение 2-го порядка на римановой поверхности $X$ - уравнение Фукса

$$
\frac{d^{2} u_{\alpha}}{d z_{\alpha}^{2}}+\frac{1}{2} r_{\alpha} u_{\alpha}=0
$$

где $U=\left\{u_{\alpha}\right\}$ понимается как многозначный дифференциал порядка $-1 / 2$ на $X$. Уравнение (2) определяет группу монодромии - представление фундаментальной группы $\pi_{1}\left(X, x_{0}\right)$ римановой поверхности $X$ с выделенной точкой $x_{0}$ в $\operatorname{PSL}(2, \mathbb{C})=$ $\mathrm{SL}(2, \mathbb{C}) /\{ \pm I\}$. Из условия (1) следует, что при отображении монодромии образы стандартных образующих в $\pi_{1}\left(X, x_{0}\right)$, отвечающих обходам вокруг проколов $x_{i}$, являются параболическими элементами в $\operatorname{PSL}(2, \mathbb{C})$.

2.2. Униформизация. Согласно теореме об униформизации

$$
X \cong \Gamma \backslash \mathbb{H}
$$

где $\mathbb{H}=\{\tau \in \mathbb{C}: \operatorname{Im} \tau>0\}-$ модель Пуанкаре плоскости Лобачевского, а $\Gamma \subset$ $\operatorname{PSL}(2, \mathbb{R})$ - фуксова группа типа $(g, n)$, действующая на $\mathbb{H}$ дробно-линейными преобразованиями. Другими словами, существует комплексно-аналитическое накрытие $J: \mathbb{H} \rightarrow X$, группой автоморфизмов которого является $\Gamma$. Обратная к $J$ многозначная аналитическая функция $J^{-1}: X \rightarrow \mathbb{H}$ представляет собой локально-однолистную, линейно-полиморфную функцию на римановой поверхности $X$ (последнее означает, что ее ветви связаны между собой дробно-линейными преобразованиями из Г). Производные Шварца функции $J^{-1}$ по $z_{\alpha}$ в $U_{\alpha}$ корректно определены и задают фуксову проективную связность $R_{\mathrm{F}}=\left\{\mathcal{S}_{z_{\alpha}}\left(J^{-1}\right)\right\}$ на $X$, а многозначные функции $1 / \sqrt{\left(J^{-1}\right)^{\prime}}$ и $J^{-1} / \sqrt{\left(J^{-1}\right)^{\prime}}$ удовлетворяют уравнению Фукса $(2)$ с $R=R_{\mathrm{F}}$. Группа монодромии этого уравнения совпадает, с точностью до сопряжения в $\operatorname{PSL}(2, \mathbb{R})$, с фуксовой группой $\Gamma$.

Клейн [1] и Пуанкаре [2] решали проблему униформизации римановой поверхности $X$, подбирая проективную связность в уравнении Фукса (2) так, чтобы его группа монодромии оказалась такой фуксовой группой Г, что выполняется соотношение (3). Прямое доказательство существования фуксовой проективной связности $R_{\mathrm{F}}$ на $X$ оказалось весьма трудной задачей, которая и по сей день не получила окончательного решения (см. [11], [12]). В случае римановых поверхностей типа $(0, n)$, которыми мы здесь ограничимся, эта задача формулируется следующим образом.

Пусть $X_{0}$ - сфера Римана $\mathbb{P}^{1}$, а $X$ - риманова поверхность рода 0 с $n$ проколами $z_{1}, \ldots, z_{n}$. Без ограничения общности можно считать, что $z_{n-2}=0, z_{n-1}=1$, 
$z_{n}=\infty$ и $X=\mathbb{C} \backslash\left\{z_{1}, \ldots, z_{n-3}, 0,1\right\}$. Уравнение (2) принимает вид

$$
\frac{d^{2} u}{d z^{2}}+\frac{1}{2} \sum_{i=1}^{n-1}\left(\frac{1}{2\left(z-z_{i}\right)^{2}}+\frac{c_{i}}{z-z_{i}}\right) u=0
$$

где $z$ - глобальная комплексная координата на $X$. Комплексные параметры $c_{1}, \ldots$, $c_{n-1}$ удовлетворяют соотношениям

$$
\sum_{i=1}^{n-1} c_{i}=0, \quad \sum_{i=1}^{n-1} z_{i} c_{i}=1-\frac{n}{2}
$$

которые позволяют явно выразить $c_{n-2}$ и $c_{n-1}$ через $z_{1}, \ldots, z_{n-3}$ и оставшиеся $n-3$ параметра $c_{1}, \ldots, c_{n-3}$.

В классическом подходе Клейна и Пуанкаре к проблеме униформизации по заданным регулярным особым точкам $z_{1}, \ldots, z_{n-3}, 0,1, \infty$ требовалось выбрать параметры $c_{1}, \ldots, c_{n-3}$ таким образом, чтобы группа монодромии уравнения (4) была фуксовой группой, изоморфной фундаментальной группе римановой поверхности $X$. Тогда отношение двух линейно независимых решений уравнения (4) и являлось бы, с точностью до дробно-линейного преобразования, искомым многозначным отображением $J^{-1}: X \rightarrow \mathbb{H}$, осуществляющим униформизацию римановой поверхности $X$. Соответствующая Г-автоморфная функция $J: \mathbb{H} \rightarrow \mathbb{C}$ называется инвариантом Клейна. Комплексные числа $c_{1}, \ldots, c_{n-3}$, акцессорнъе параметры фуксовой униформизации поверхности $X$, однозначно определяются по особым точкам $z_{1}, \ldots, z_{n-3}$. При этом

$$
\mathcal{S}\left(J^{-1}\right)(z)=\sum_{i=1}^{n-1}\left(\frac{1}{2\left(z-z_{i}\right)^{2}}+\frac{c_{i}}{z-z_{i}}\right) .
$$

Для доказательства существования акцессорных параметров Пуанкаре предложил так называемый метод непрерьвности [2]. Однако этот метод не позволил получить строгое решение проблемы униформизации и, подвергнутый критике, был вскоре оставлен. Окончательное решение проблемы униформизации было получено Кёбе и Пуанкаре в 1907 г. при помощи принципиально иных методов, в частности с использованием теории потенциала (современное изложение см., например, в [13]).

2.3. Уравнение Лиувилля. Проекция на $X$ метрики Пуанкаре $(\operatorname{Im} \tau)^{-2}|d \tau|^{2}$ на $\mathbb{H}$ представляет собой полную метрику постоянной отрицательной кривизны -1 , согласованную с комплексной структурой на $X$. Она имеет вид $e^{\varphi(z)}|d z|^{2}$, где

$$
e^{\varphi(z)}=\frac{\left|\left(J^{-1}\right)^{\prime}(z)\right|^{2}}{\left(\operatorname{Im} J^{-1}(z)\right)^{2}}
$$

Гладкая функция $\varphi$ на $X$ удовлетворяет уравнению Лиувилля

$$
\varphi_{z \bar{z}}=\frac{1}{2} e^{\varphi}
$$

и имеет следующие асимптотики:

$$
\varphi(z)= \begin{cases}-2 \ln \left|z-z_{i}\right|-2 \ln |\ln | z-z_{i} \mid+o(1), & z \rightarrow z_{i}, \quad i \neq n, \\ -2 \ln |z|-2 \ln \ln |z|+o(1), & z \rightarrow \infty\end{cases}
$$

8 Теоретическая и математическая физика, т. 181, № 1, 2014 г. 
В работе [3] Пуанкаре предложил подход к проблеме униформизации, использующий уравнение Лиувилля. А именно, он доказал, что в классе гладких вещественнозначных функций на $X$ с асимптотиками (9) уравнение Лиувилля (8) однозначно разрешимо. Отсюда следует, что $T_{\varphi}=\varphi_{z z}-\varphi_{z}^{2} / 2$ является рациональной функцией вида (6), а уравнение

$$
\frac{d^{2} u}{d z^{2}}+\frac{1}{2} T_{\varphi} u=0
$$

имеет фуксову группу монодромии, которая и униформизует риманову поверхность $X$.

Уравнение Лиувилля является уравнением Эйлера-Лагранжа для экстремалей функционала

$$
S(\psi)=\lim _{\varepsilon \rightarrow 0}\left(\iint_{X_{\varepsilon}}\left(\left|\psi_{z}\right|^{2}+e^{\psi}\right) d^{2} z+2 \pi n \ln \varepsilon+4 \pi(n-2) \ln |\ln \varepsilon|\right),
$$

где $d^{2} z$ - мера Лебега на $\mathbb{C}$,

$$
X_{\varepsilon}=X \backslash\left(\bigcup_{i=1}^{n-1}\left\{\left|z-z_{i}\right|<\varepsilon\right\} \cup\left\{|z|>\frac{1}{\varepsilon}\right\}\right),
$$

а $\psi$ принадлежит классу функций с асимптотиками (9). Величина $T_{\psi}=\psi_{z z}-\psi_{z}^{2} / 2$ играет роль $(2,0)$-компоненты тензора энергии-импульса в классической теории Лиувилля и

$$
T_{\varphi}=\mathcal{S}\left(J^{-1}\right)
$$

Обозначим через

$$
\mathcal{M}_{0, n}=\left\{\left(z_{1}, \ldots, z_{n-3}\right) \in \mathbb{C}^{n-3}: z_{i} \neq z_{j} \text { для } i \neq j, \text { и } z_{i} \neq 0,1\right\}
$$

пространство модулей римановых поверхностей рода 0 с $n$ упорядоченными проколами (рациональных кривых с $n$ отмеченными точками). Критические значения функционала действия уравнения Лиувилля $S(\psi)$ (значения на экстремалях $\varphi$ для всех поверхностей $X$ ) задают гладкую функцию $S: \mathcal{M}_{0, n} \rightarrow \mathbb{R}-$ классическое действие уравнения Лиувилля. Как было показано в работах [5], [6], классическое действие уравнения Лиувилля играет фундаментальную роль в комплексной геометрии пространства модулей $\mathcal{M}_{0, n}$. А именно, $S$ является общей первообразной функцией для акцессорных параметров

$$
c_{i}=-\frac{1}{2 \pi} \frac{\partial S}{\partial z_{i}}, \quad i=1, \ldots, n-3,
$$

и кэлеровым потенциалом метрики Вейля-Петерсона на $\mathcal{M}_{0, n}$,

$$
-\frac{\partial^{2} S}{\partial z_{i} \partial \bar{z}_{j}}=\left\langle\frac{\partial}{\partial z_{i}}, \frac{\partial}{\partial z_{j}}\right\rangle_{W P}, \quad i, j=1, \ldots, n-3 .
$$

Утверждение, что для поверхностей рода 0 классическое действие уравнения Лиувилля является производящей функцией для акцессорных параметров, было высказано Поляковым ${ }^{1)}$ на основе анализа квазиклассического предела конформных тождеств Уорда квантовой теории Лиувилля (см. [14]).

1) Доклад в Ленинградском отделении Математического института им. В. А. Стеклова, 1982 г., не опубликовано. 


\section{3. ВЕЩЕСТВЕННЫЕ ПРОЕКТИВНЫЕ СВЯЗНОСТИ И ДИССЕРТАЦИЯ В.И. СМИРНОВА}

3.1. Общий случай. Пусть $X$ - риманова поверхность типа $(g, n)$. Проективная связность $R$ на $X$ называется вещественной (фуксовой), если ее группа монодромии, с точностью до сопряжения в $\operatorname{PSL}(2, \mathbb{C})$, есть подгруппа в $\operatorname{PSL}(2, \mathbb{R})$ (фуксова группа). Согласно теореме об униформизации фуксова проективная связность $R_{\mathrm{F}}$ однозначно характеризуется условием, что ее группа монодромии в точности есть фуксова группа $\Gamma$, униформизирующая поверхность $X$. Естественно возникает вопрос, нельзя ли задать проективную связность $R_{\mathrm{F}}$ на поверхности $X$ более простыми условиями типа вещественности (см. стр. 224 в работе [15]) или фуксовости? Ответ на этот вопрос отрицателен.

Именно, в случае, когда $X=\Gamma \backslash \mathbb{H}$ есть компактная риманова поверхность рода $g>1$, Голдман [7] показал, что каждой целочисленной измеримой ламинации Тёрстона $\mu=\sum_{i} m_{i} \gamma_{i}$ (дизъюнктное объединение), где $\gamma_{i}$ - простые замкнутые геодезические в гиперболической метрике на $X$, а $m_{i}$ - неотрицательные целые числа, отвечает риманова поверхность $G r_{\mu}(X)$ рода $g$ с проективной связностью $R(\mu)$, имеющей группу монодромии Г. Римановы поверхности $G r_{\mu}(X)$ получаются из $X$ при помощи так называемой процедуры "графтинга" и обобщают классические примеры Маскита-Хейхала и Салливана-Тёрстона (см. [7]). При этом множество фуксовых проективных связностей на всех римановых поверхностях рода $g$ изоморфно прямому произведению пространства Тейхмюллера $T_{g}$ и множества целочисленных измеримых ламинаций на топологической поверхности рода $g$. В работе [16] доказано, что на каждой римановой поверхности $X$ рода $g>1$ существует бесконечно много проективных связностей с фуксовой монодромией.

Вещественные проективные связности для римановых поверхностей типа $(g, n)$ также рассматривались Фальтингсом [15]. Как показано в работе [7] для компактных римановых поверхностей, каждой полуцелой измеримой ламинации Тёрстона $\mu$ отвечает риманова поверхность $G r_{\mu}(X)$ рода $g$ с вещественной проективной связностью.

3.2. Поверхности типа $(0,4)$ и подход В. И. Смирнова. Классики связывали задачу об униформизации римановых поверхностей с дифференциальными уравнениями. В качестве основного примера рассматривался случай римановых поверхностей типа $(0,4)$, и ставилась задача о нахождении такого акцессорного параметра в уравнении (4), чтобы его группа монодромии была фуксовой или клейновой (дискретной подгруппой $\operatorname{PSL}(2, \mathbb{C}))$. Для специального случая вещественных особых точек Клейн [17] предложил подход, в котором используется осцилляционная теорема типа Штурма, а Хилб [18] доказал, что уравнение (4) имеет фуксову группу монодромии для бесконечного числа значений акцессорного параметра. Гильберт [19] свел эту задачу к исследованию некоторого интегрального уравнения.

Полностью задача о вещественной группе монодромии уравнения (4) с четырьмя вещественными особыми точками была решена в магистерской диссертации В. И. Смирнова [8], изданной в Петрограде в 1918 г. (ее основное содержание приведено в [9], [10]). Именно, рассмотрим уравнение (4) с особыми точками $z_{1}=0$, $z_{2}=a, z_{3}=0$ и $z_{4}=\infty$, где $0<a<1$. Записывая общее решение уравнений $(5)$ в виде

$$
c_{1}=1+\frac{1+2 \lambda}{a}, \quad c_{2}=\frac{1+2 \lambda}{a(a-1)}, \quad c_{3}=-\frac{a+2 \lambda}{a-1}
$$


где $\lambda$ - акцессорный параметр, и совершая замену зависимой переменной $y=$ $\sqrt{z(z-a)(z-1)} u$, приходим к уравнению

$$
\frac{d}{d z}\left(p(z) \frac{d y}{d z}\right)+(z+\lambda) y=0, \quad p(z)=z(z-a)(z-1)
$$

Обозначим через $\left(y_{i}^{(1)}, y_{i}^{(2)}\right)$ стандартный базис пространства решений уравнения (10) в окрестности каждой из особых точек $z_{i}$, состоящий из нормированных голоморфных решений

$$
\begin{aligned}
& y_{i}^{(1)}(z, \lambda)=1+\sum_{k=1}^{\infty} a_{i k}\left(z-z_{i}\right)^{k}, \quad i=1,2,3 \\
& y_{4}^{(1)}(z, \lambda)=\frac{1}{z}+\sum_{k=2}^{\infty} \frac{a_{4 k}}{z^{k}}
\end{aligned}
$$

и решений

$$
\begin{aligned}
& y_{i}^{(2)}(z, \lambda)=y_{i}^{(1)}(z, \lambda) \ln \left(z-z_{i}\right)+\tilde{y}_{i}(z, \lambda), \quad i=1,2,3 \\
& y_{4}^{(2)}(z, \lambda)=y_{4}^{(1)}(z, \lambda) \ln \frac{1}{z}+\tilde{y}_{4}(z, \lambda)
\end{aligned}
$$

где $\tilde{y}_{i}(z, \lambda)$ голоморфны в окрестности $z_{i}$. При вещественных $\lambda$ степенные ряды $y_{i}^{(1,2)}(z, \lambda)$ и $\widetilde{y}_{i}(z, \lambda)$ имеют вещественные коэффициенты.

Для определения вещественных значений $\lambda$, при которых группа монодромии уравнения (10) вещественна, классики использовали понятие вещественного продолжения. А именно (см. [8]), если в окрестности особой точки $a$ при вещественных $z<a$ мы имеем

$$
y(z)=c \ln (a-z)+f(z),
$$

где $\ln 1=0$ и функция $f(z)$ голоморфна в окрестности $a$, то вещественное продолжение $y$ в область $z>a$ определяется как

$$
y(z)=c \ln (z-a)+f(z) .
$$

Справедливо следующее утверждение.

Теорема 1 (Клейн, Гильберт). Уравнение (10) имеет вещественную группу монодромии, если $\lambda$ вещественно и выполняется одно из следующих условий:

1) решение $y_{0}^{(1)}(z, \lambda)$ голоморфно в окрестности особой точки $z_{2}=a$;

2) решение $y_{2}^{(1)}(z, \lambda)$ голоморфно в окрестности особой точки $z_{3}=1$;

3) при вещественном продолюении через $z=$ а решение $y_{0}^{(1)}(z, \lambda)$ голоморфно в окрестности особой точки $z_{3}=1$.

При этом в случае 1 отношение $\eta=\sqrt{-1} y_{3}^{(1)} / y_{1}^{(1)}$ линейно независимых решений уравнения (10) при обходе вокруг особых точек $0, a$ и 1 преобразуется при помощи вещественных дробно-линейных преобразований; в случае 2 аналогичную роль играет отношение $\eta=\sqrt{-1} y_{2}^{(1)} / y_{4}^{(1)}$, а в случае 3 - отношение $\eta=\sqrt{-1} y_{1}^{(1)} / y_{2}^{(1)}$ (подробнее см. [8]).

Условия 1-3 определяют следующие три спектральные задачи типа ШтурмаЛиувилля для уравнения (10). Именно, требуется найти те значения $\lambda$, при которых 


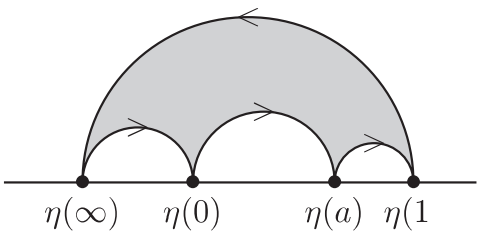

Рис. 1

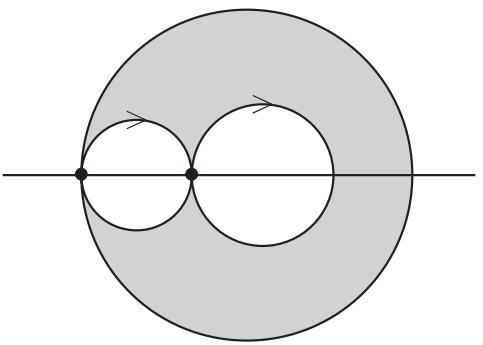

Рис. 2

1) на отрезке $[0, a]$ существует решение, регулярное в точках 0 и $a$;

2) на отрезке $[a, 1]$ существует решение, регулярное в точках $a$ и 1 ;

3) существует решение, регулярное в точке 0 и такое, что при вещественном продолжении через точку $а$ оно регулярно и в точке 1.

Используя классический метод Штурма, В.И. Смирнов [8] доказал следующее утверждение.

Теорема 2 (В.И. Смирнов). Каждая из задач Штурма-Лиувилля 1-3 имеет простой неограниченный дискретный спектр. Справедливы следующие утверждения.

1. Спектральная задача 1 имеет бесконечно много собственных значений $\mu_{k}$, $k \in \mathbb{N}$, сгущающихся $\kappa \infty$ и удовлетворяющих неравенствам

$$
-a<\mu_{1}<\mu_{2}<\ldots
$$

2. Спектральная задача 2 имеет бесконечно много собственных значений $\mu_{-k}$, $k \in \mathbb{N}$, сгущающихся $\kappa-\infty$ и удовлетворяющих неравенствам

$$
-a>\mu_{-1}>\mu_{-2}>\ldots
$$

3. Спектральная задача 3 имеет бесконечно много собственных значений $\lambda_{k}$, $k \in \mathbb{Z}$, сгущающихся $\kappa \pm \infty$ и удовлетворяющих неравенствам

$$
\cdots<\mu_{-2}<\lambda_{-1}<\mu_{-1}<\lambda_{0}<\mu_{1}<\lambda_{1}<\mu_{2}<\ldots
$$

Случай $\lambda=\lambda_{0}$ отвечает фуксовой униформизации римановой поверхности $X=$ $\mathbb{C} \backslash\{0, a, 1\}$, и отношение $\eta=\sqrt{-1} y_{1}^{(1)} / y_{2}^{(1)}$ взаимно однозначно отображает верхнюю полуплоскость переменной $z$ на внутренность кругового четырехугольника с нулевыми углами и сторонами, ортогональными $\mathbb{R} \cup\{\infty\}$. Нормируя $\eta$ при помощи вещественного дробно-линейного преобразования так, чтобы образы особых точек $0, a, 1$ и $\infty$ были конечны, получаем четырехугольник, изображенный на рис. 1 (ср. с рис. 9 в [17]). Аналитически продолжая $\eta(z)$ в нижнюю полуплоскость переменной $z$, получаем многозначную линейно-полиморфную функцию $\eta: X \rightarrow \mathbb{H}$ с фуксовой группой Г такую, что $J=\eta^{-1}$ задает изоморфизм (3).

Соответствующее отношение $\eta$ будет взаимно однозначной функцией на верхней полуплоскости переменной $z$ и в случаях $\lambda=\mu_{ \pm 1}$. Так, при $\lambda=\mu_{1}$ имеем $\eta(0)=\eta(a)=\infty$ и $\eta(1)=\eta(\infty)=0$. Нормируя $\eta=\sqrt{-1} y_{3}^{(1)} / y_{1}^{(1)}$ так, чтобы образы 


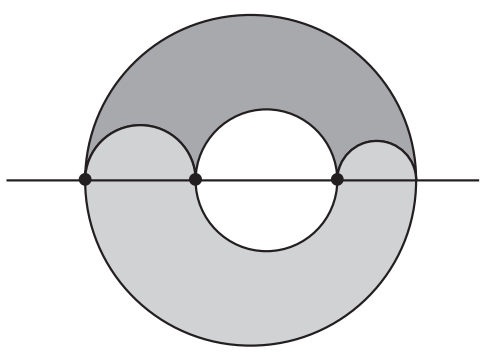

Рис. 3

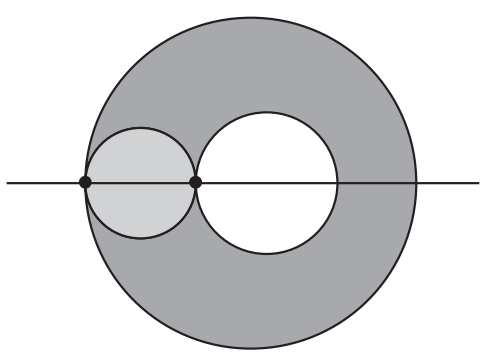

Рис. 4

особых точек были конечны, получаем, что $\eta$ взаимно однозначно отображает верхнюю полуплоскость $z$ на внутренность вырожденного кругового четырехугольника, изображенного на рис. 2. Соответствующая группа монодромии будет группой типа Шоттки.

При всех остальных значениях $\lambda_{k}$ и $\mu_{k}$ соответствующее отображение $\eta$ уже не взаимно однозначно в верхней полуплоскости переменной $z$. Так, при $\lambda=\lambda_{1}$ верхняя полуплоскость отображается на внутренность кругового кольца, изображенного на рис. 3 (ср. с рис. 10 в [17]). При этом функция $\eta$ принимает по два раза значения из отмеченной темным цветом области, которая отвечает четырехугольнику на рис. 1. При $\lambda=\lambda_{k}$ этот четырехугольник наматывается на себя $2|k|$ раз.

Аналогичным образом при $\lambda=\mu_{2}$ верхняя полуплоскость переменной $z$ отображается на внутренность кругового кольца, изображенного на рис. 4. При этом функция $\eta$ принимает по два раза значения из отмеченной темным цветом области, которая отвечает вырожденному четырехугольнику на рис. 2 . При $\lambda=\mu_{k}$ этот четырехугольник наматывается на себя $|k|$ раз.

Поучительно сравнить эти результаты В. И. Смирнова с классификацией Голдмана фуксовых и вещественных проективных связностей на римановых поверхностях, обобщенной на случай поверхностей типа $(g, n)$. Мы получаем, что фуксова серия $\lambda=\lambda_{k}$ отвечает целочисленным ламинациям в [7], а серия $\lambda=\mu_{k}-$ полуцелым ламинациям.

\section{4. РЕШЕНИЯ УРАВНЕНИЯ ЛИУВИЛЛЯ ТИПА ЧЕРНЫХ ДЫР}

Фуксова униформизация римановой поверхности $X$ определяет решение уравнения Лиувилля - гладкую функцию $\varphi$ на $X$, удовлетворяющую уравнению (8) и имеющую асимптотики (9) (см. п. 2.3). Функция $\varphi$ определяется по отношению $J^{-1}$ линейно независимых решений уравнения (4) по формуле (7). Корректность этой формулы обеспечивается условием вещественности группы монодромии Г уравнения (4), а ее гладкость - условием, что $Г$ униформизует риманову поверхность $X$ и ее образ при многозначном отображении $J^{-1}$ есть верхняя полуплоскость $\mathbb{H}$.

Аналогичным образом с каждым уравнением (4) с вещественной группой монодромии можно связать решение уравнения Лиувилля. Положим

$$
e^{\varphi(z)}=\frac{\left|\eta^{\prime}(z)\right|^{2}}{(\operatorname{Im} \eta(z))^{2}},
$$


где $\eta$ - отношение линейно независимых решений уравнения (4), преобразующееся при помощи вещественных дробно-линейных преобразований при обходе вокруг особых точек (в фуксовом случае $\eta=J^{-1}$ ). Функция $\varphi$ корректно определена благодаря вещественности монодромии и имеет асимптотики (9). Последнее следует из теории фуксовых уравнений с одинаковыми показателями. Однако решение (11) уже не является гладким: образ $X$ при многозначном отображении $\eta$ имеет нетривиальное пересечение с вещественной осью и функция $\varphi$ сингулярна на $\eta^{-1}(\mathbb{R})$.

Именно, из результатов [15] (см. §6 в указанной работе) следует, что полный прообраз $\eta^{-1}(\mathbb{R})$ есть дизъюнктное объединение конечного числа простых замкнутых аналитических кривых на $X$. Пусть $C$ - одна из таких кривых. Существует ветвь многозначной функции $\eta$, взаимно однозначно отображающая $C$ на окружность, так что $C=\left\{z=\eta^{-1}(t), t \in[\alpha, \beta]\right\}$. Удобно ввести функцию Швариа $S$ аналитического контура $C$ по формуле

$$
S=\bar{\eta}^{-1} \circ \eta
$$

где $\bar{\eta}^{-1}(z)=\overline{\eta^{-1}(\bar{z})}$. Функция Шварца определена в некоторой окрестности контура $C$ и задает его уравнением $\bar{z}=S(z)$ (см. [20]). Несложно показать, что в терминах функции Шварца решение $\varphi$ имеет те же сингулярности на $C$, что и функция

$$
-\frac{4 \overline{S^{\prime}(z)}}{(z-\overline{S(z)})^{2}}
$$

Именно, при $z \rightarrow z_{0} \in C$ вдоль любого не касательного к $C$ направления

$$
e^{\varphi(z)}=-\frac{4 S^{\prime}\left(z_{0}\right)}{\left(\bar{z}-\bar{z}_{0}-S^{\prime}\left(z_{0}\right)\left(z-z_{0}\right)\right)^{2}}\left(1+O\left(\left|z-z_{0}\right|\right)\right) .
$$

Отметим, что в силу условия $\bar{S}(S(z))=z$ функция в правой части формулы (13) вещественна и положительна. Сингулярности типа $(12),(13)$ на контуре $C$ аналогичны сингулярности на $\mathbb{R}$ метрики Пуанкаре на $\mathbb{C} \backslash \mathbb{R}$, отвечающей функции Шварца $S(z)=z$.

В результате мы приходим к следующей задаче. На римановой поверхности $X=\mathbb{C} \backslash\left\{z_{1}, \ldots, z_{n-3}, 0,1\right\}$ требуется найти такие простые аналитические контуры $C_{1}, \ldots, C_{k}$ и функцию $\varphi$, что на $X \backslash \bigcup_{j=1}^{k} C_{j}$ функция $\varphi$ удовлетворяет уравнению Лиувилля (8), имеет асимптотики (9) в проколах $z_{i}$ и сингулярности типа (12), (13) на контурах $C_{j}$. На каждой из компонент связности $X e^{\varphi(z)}|d z|^{2}$ задает полную метрику постоянной отрицательной кривизны -1 . Границу $C_{j}$ можно интерпретировать как горизонт черной дыры, поэтому соответствующие решения уравнения Лиувилля мы называем решениями тuпа черньх дыр. Из классификации Голдмана [7] вещественных проективных связностей следует существование семейства таких решений, параметризованных "целочисленной решеткой" целых и измеримых ламинаций Тёрстона и неявно задаваемых процедурой графтинга.

Из результатов диссертации В. И. Смирнова следует явное описание решений типа черных дыр для случая четырех вещественных проколов. Именно, из теоремы 2 получаем следующее утверждение. 
ТЕОРема 3. Все решения уравнения Лиувилля типа черных дыр для случая четырех вещественных проколов $0, a, 1$ и описываются следующим образом.

1. Решения фуксового типа, отвечающие значениям акцессорного параметра $\lambda=\lambda_{k}, k$ - целое число, и имеющие $2|k|$ контуров $C_{j}$. При $k>0$ эти контуры обходят точки 0 и а, а при $k<0$ - точки а и 1 .

2. Решения типа Шоттки, отвечающие значениям акцессорного параметра $\lambda=\mu_{k}, k \neq 0$ - иелое число, и имеющие $2|k|-1$ контуров $C_{j}$. При $k>0$ эти контуры обходят точки 0 и а, а при $k<0$ - точки а и 1.

В общем случае удобно использовать подстановку $\chi(z)=e^{-\varphi(z) / 2}$, приводящую уравнение Лиувилля (8) к виду

$$
-\chi \chi_{z \bar{z}}+\left|\chi_{z}\right|^{2}=\frac{1}{4}
$$

а асимптотики (9) - к виду

$$
\chi(z)= \begin{cases}\left|z-z_{i}\right||\ln | z-z_{i}||(1+o(1)), & z \rightarrow z_{i}, \quad i \neq n, \\ |z| \ln |z|(1+o(1)), & z \rightarrow \infty .\end{cases}
$$

Сингулярности типа (12) переходят в условие обращение в нуль на контуре $C$ :

$$
\chi(z) \sim \frac{z-\overline{S(z)}}{2 \sqrt{-\overline{S^{\prime}(z)}}}
$$

так что при отражении Шварца $z^{*}=S(z)$ через контур $C$ вещественная функция $\chi(z)$ меняет знак. Эллиптическое уравнение в частных производных (14) с асимптотиками (15) и условиями вырождения (16) на контурах $C_{j}$ представляет собой краевую задачу со свободной границей. Для ее решения интересно использовать метод продолжения по параметру с использованием априорной оценки, примененный в [3] к уравнению Лиувилля (8) с асимптотиками (9).

В заключение отметим, что функция $\chi$ играет важную роль в теории уравнения Лиувилля. А именно, она представляется как билинейная форма от решений уравнения (4) их комплексно-сопряженных и удовлетворяет (4)

$$
\chi_{z z}+\frac{1}{2} T_{\varphi} \chi=0
$$

и комплексно-сопряженному уравнению. В квантовой теории Лиувилля поле $\chi=$ $e^{-\varphi(z) / 2}$ описывает вырожденный на уровне 2 вектор в модуле Верма алгебры Вирасоро. В случае решений типа черных дыр функция $\chi$ по-прежнему удовлетворяет уравнению (17). Было бы интересно выяснить, какую роль она играет в квантовой теории Лиувилля.

Благодарности. Автор благодарен П. Г. Зографу за полезные обсуждения работ [7], [15] и геометрическую интерпретацию результатов диссертации В. И. Смирнова. Приведенные результаты частично докладывались на конференциях "Perspectives, Open Problems \& Applications of Quantum Liouville Theory" в Стони Бруке (США, март 2010 г.) и "Математика - XXI век. 70 лет ПОМИ" (Санкт-Петербург, сентябрь 2010 г.). Работа выполнена при поддержке Национального научного фонда (NSF, гранты № DMS-0705263 и DMS-1005769). 


\section{Список литературы}

[1] F. Klein, Math. Ann., 21:2 (1883), 141-218.

[2] H. Poincaré, Acta Math., 4:1 (1884), 201-312; А. Пуанкаре, "О группах линейных уравнений”, Избранные трудь, т. 3: Математика. Теоретическал физика. Анализ математических и естественнонаучных работ Анри Пуанкаре, Наука, М., 1974, 145-234.

[3] H. Poincaré, J. Math. Pures Appl., 4 (1898), 157-230; А. Пуанкаре, Избраннъье трудъ, т. 3: Математика. Теоретическая физика. Анализ математических и естественнонаучных работ Анри Пуанкаре, 3, Наука, М., 1974.

[4] А.Н. Тюрин, УМН, 33:6(204) (1978), 149-195.

[5] П. Г. Зограф, Л. А. Тахтаджян, Функи. анализ и его прил., 19:3 (1985), 67-68.

[6] П. Г. Зограф, Л. А. Тахтаджян, Матем. сб., 132(174):2 (1987), 147-166.

[7] W. M. Goldman, J. Diff. Geom., 25 (1987), 297-326.

[8] В.И. Смирнов, Задача обращения линейного дифференциального уравнения второго порядка с четыръмя особыми точками, Дис. ... магистра, Петроградский университет, Петроград, 1918; Избранные труды: Аналитическая теория обыкновенных дифференциальных уравнений, Изд-во СПбГУ, СПб., 1996, 9-211.

[9] V. Smirnov, C. R. Sci. Acad. Paris, 171 (1920), 510-512; В. И. Смирнов, Избраннъце труды: Аналитическая теория обыкновенных дифференциальных уравнений, Изд-во СПбГУ, СПб., 1996, 212-214.

[10] V. Smirnov, Bull. Sci. Math., 45 (1921), 93-120; 126-135; В. И. Смирнов, Избранные труды: Аналитическая теория обыкновенных дифференииальных уравнений, Изд-во СПбГУ, СПб., 1996, 215-249.

[11] D. Hejhal, Acta Math., 135:1 (1975), 1-55.

[12] D. Hejhal, Bull. AMS, 84:3 (1978), 339-376.

[13] H. M. Farkas, I. Kra, Riemann Surfaces, Graduate Texts in Mathematics, 71, Springer, New York, 1992.

[14] L. A. Takhtajan, "Topics in the quantum geometry of Riemann surfaces: two-dimensional quantum gravity", Quantum Groups and Their Applications in Physics (Varenna, 1994), Proceedings of the International School of Physics "Enrico Fermi", 127, eds. L. Castellani, J. Wess, IOS Press, Amsterdam, 1996, 541-579.

[15] G. Faltings, Compos. Math., 48:2 (1983), 223-269.

[16] H. Tanigawa, J. Diff. Geom., 47:3 (1997), 399-419.

[17] F. Klein, Math. Ann., 64:2 (1907), 175-196.

[18] E. Hilb, Math. Ann., 66 (1909), 215-257.

[19] D. Hilbert, Grudzüge der allgemeinen Theorie der linearen Integralgleichungen, Chelsea, New York, 1953.

[20] P. J. Davis, The Schwarz function and its applications, The Carus Mathematical Monographs, 17, MAA, Buffalo, NY, 1974. 